\title{
Serum Human Epididymis Protein 4 Level is Associated with Cognitive Function in Patients with Diabetes Mellitus
}

This article was published in the following Dove Press journal: Diabetes, Metabolic Syndrome and Obesity: Targets and Therapy

\author{
Fengfeng Bai ${ }^{1} *$ \\ Tao $\mathrm{Li}^{1, *}$ \\ Baozhu Li' \\ Xiaozheng $\mathrm{Li}^{1}$ \\ Lifeng Zhu ${ }^{2}$ \\ 'Department of Psychiatry, Anding \\ Hospital, Tianjin 300222, People's \\ Republic of China; ${ }^{2}$ Nursing Department, \\ The Second Hospital, Cheeloo College of \\ Medicine, Shandong University, Jinan, \\ Shandong 250033, People's Republic of \\ China
}

*These authors contributed equally to this work
Correspondence: Lifeng Zhu

Nursing Department, The Second Hospital, Cheeloo College of Medicine, Shandong

University, 247 Beiyuan Street, Jinan,

Shandong 250033, People's Republic of

China

Tel +86 I7660087098

Email28I4539930@qq.com
Background: Previous studies have reported that patients with diabetes mellitus (DM) have a higher incidence of cognitive decline and an increased risk of developing all types of dementia. The aim was to elucidate the association between serum human epididymal protein 4 and cognitive function in patients with DM.

Methods: Serum levels of HE4 were measured in 205 patients with DM. All DM patients were followed up for a median period of 48 months (range=5-49) prospectively. Cox proportional hazard analysis was used to evaluate the predictive value of serum HE4 for predicting cognitive decline (end point).

Results: Multivariate linear regression analysis revealed that serum HE4 was independently associated with MOCA score after adjusting for age, gender, BMI, current smoker, current drinker, admission systolic and diastolic BP, CVD history and laboratory measurements in patients with $\mathrm{DM}$ at baseline $(\mathrm{S} \beta=-0.120 ; 95 \% \mathrm{CI},-0.151--0.069 ; P<0.001)$. The multivariate Cox proportional hazard analysis revealed that serum HE4 (HR=2.408, 95\% CI 1.669-5.238, $P<0.001)$ was an independent prognostic factor for cognitive decline in these DM patients.

Conclusion: Our results showed that serum HE4 was significantly and independently associated with cognitive decline and had independent predictive value for cognitive decline in patients with DM. Serum HE4 might enable early recognition of senile dementia among DM patients.

Keywords: human epididymis protein 4, diabetes mellitus, cognitive decline, prognostic value

\section{Introduction}

Persons with diabetes have been reported to hold a higher incidence of cognitive decline and senile dementia, diabetes mellitus (DM) has been strongly associated with an increased risk of developing all types of senile dementia. ${ }^{1-4}$ Existing studies suggested that in most studies the incidence of "any dementia" was higher in persons with diabetes than in those without diabetes..$^{5}$ There are methodological limitations in some studies, but the association remains strong. Although the precise mechanisms are currently poorly understood, increasing inflammation and oxidative stress in DM play important role in causing an increased risk for cognitive decline. ${ }^{6}$ The early prediction of cognitive decline in patients with DM might provide an opportunity to develop strategies to improve prognosis.

Human epididymis protein 4 (HE4), encoded by the WFDC2 gene located on chromosome 20q12-13.1, is a secretory protein highly expressed in the human 
epididymis. ${ }^{7}$ The HE4 sequence shows similarity to proteinase inhibitors, which suggests that HE4 may be involved in sperm maturation. ${ }^{8}$ The mature HE4 protein is a $20-25 \mathrm{kDa}$ glycoprotein found in the cytoplasm and on membranes of cells and in circulation. Studies have reported that HE4 is highly expressed in malignant tumors such as ovarian cancer and endometrial cancer tumors. ${ }^{9,10}$ Additionally, some studies have shown that HE4 is moderately expressed in multiple normal and abnormal tissues in the human body, such as the respiratory tract, convoluted tubules of the kidney, and other tissues, ${ }^{11,12}$ and it plays a very important role in process related to immune defense. Other WFDC proteins with antiproteinase activity have also been correlated with inflammatory processes. ${ }^{13-15}$ It has been widely recognized that inflammation is involved in the occurrence and development of cognitive decline. ${ }^{16,17}$ As a chronic inflammatory disease, DM can hasten the progression of cognitive decline and contribute to a higher rate of senile dementia. $^{18,19}$

Given the close association between HE4 and inflammation, ${ }^{13-15}$ we hypothesized that HE4 may be associated with cognitive decline in DM patients. However, to date, no relevant study has explored the relationship between serum HE4 and cognitive decline in patients with DM. The aim of this study was to investigate whether increased HE4 levels contribute to the increased risk for cognitive decline, independent of confounding factors. This was also the first study to explore the prognostic value of HE4 for predicting cognitive function in DM patients.

\section{Materials and Methods Study Population}

We studied 205 hospitalized DM patients from Anding Hospital and The Second Hospital of Shandong University in China because of DM between January 2015 and December 2016. All DM patients were stable for three months without other serious acute illnesses before admission. After standardized hospital treatment, all DM patients were clinically stable during hospitalization. All DM patients were prospectively followed up after discharge. The diagnosis of DM was performed by two endocrinologists who used the same diagnostic criteria for these patients. ${ }^{20}$ Montreal Cognitive Assessment (MOCA) was used for the treatment of mild cognitive impairment (MCI) is a rapid screening assessment tool. The cognitive areas of assessment include attention and concentration, executive function, memory, language, visual structure skills, abstract thinking, calculation and orientation. The total score of the scale is 30 points, and patients with a value of $<26$ points were considered as MCI. Of these patients, 195 had a history of DM treatment. Patients with neoplastic diseases, or other serious diseases were excluded. Seven patients were excluded from the study because of severe liver or lung diseases or ovarian cancer or other malignant diseases.

The diagnosis of cardiovascular diseases (CVDs), including coronary heart disease, hypertension, stroke and others was based on the patient's current or previous medical records. Other data on clinical characteristics, including age, gender, body mass index (BMI), current smoking status, current alcohol consumption status, blood pressure, were collected from patient interviews or medical records. According to the Declaration of Helsinki guidelines, the Ethics Committee of Anding Hospital and the Second Hospital of Shandong University approved this study, and all patients gave written informed consent.

\section{Follow-Up}

All patients were followed up for a median period of 48 months (range $=5-49$ ) prospectively. Patients were followed up by telephone or review of the medical record three times a year until the occurrence of MCI (end points).

\section{Measurement of HE4}

Fasting venous blood samples from DM patients were obtained in the early morning within the first 24 hours after admission. The samples were prepared immediately by centrifugation and processed for determination of HE4 levels. Serum HE4 levels were tested by electrochemiluminescence immunoassay (Cobas e 601, F. Hoffmann-La Roche Ltd.). Samples with HE4 levels over $1500 \mathrm{pmol} / \mathrm{L}$ were tested again (coefficient of variation of precision $[\mathrm{CV}],<5 \%$; measurement range, $15-1500 \mathrm{pmol} / \mathrm{L}$; detection limit, $5 \mathrm{pmol} / \mathrm{L}$ ).

\section{Laboratory Measurements}

The venous blood samples drawn from the DM patients in the early morning within the first 24 hours after admission were also tested for hemoglobin $(\mathrm{Hb})$, fasting blood glucose (FBG), HbAlc (glycosylated hemoglobin), high-density lipoprotein (HDL), LDL (low-density lipoprotein), hsCRP (hs-C-reactive protein) and albumin (ALB) with the use of the Siemens ADVIA 2400 automatic biochemistry 
analyzer (Siemens AG). The Modification of Diet in Renal Disease (MDRD) formula was used to calculate estimated glomerular filtration rate (eGFR). ${ }^{21}$

\section{Statistical Analyses}

The normality of the data was analyzed by the Kolmogorov-Smirnov test combined with Q-Q plots. The data that were not normally distributed were expressed as the median (interquartile range $[\mathrm{IQR}]$ ) and analyzed by the Mann-Whitney $U$-test. Data are presented as the mean $\pm \mathrm{SD}$ for normally distributed data and were analyzed by independent $t$-test. Categorical variables were analyzed by the chi-square test. Multivariate linear regression analysis was performed to identify the independent association between serum HE4 levels and cognitive function (MOCA score) in DM patients at baseline. The Cox proportional hazard model was used to identify the independent prognostic factors for cognitive decline in patients with DM. The factors $(\mathrm{P}<0.05)$ by univariate analysis were entered into the Cox proportional hazard analyses. In addition, we also adjusted for clinical data relevant to $\mathrm{DM}$ and cognitive function even if the factors were not significantly associated with cognitive decline in the univariate analysis because they are key clinical variables and may be associated with cognitive decline in the multivariate but not univariate analyses. All of the analyses were performed by using SPSS 22.0. $P \leq 0.05$ was considered to be statistically significant.

\section{Results}

\section{Clinical Characteristics of Study Subjects at Baseline ( $\mathrm{N}=205)$}

In order to evaluate the serum levels of HE4 in DM patients, 196 age-and gender-matched subjects, who have undergone physical examination without DM and any other severe illnesses, were selected as the control group. Our results showed that serum HE4 levels were significantly higher compared with control subjects (Table 1).

The clinical characteristics of patients with DM are presented in Table 2. The age of all DM patients was
$65.6(60.3-76.0)$ years and males were $99(48.3 \%)$ MOCA score was $25.4 \pm 3.2$ in patients with DM.

\section{Serum HE4 Showed an Independent Association with MOCA Score in 205 Patients with DM}

To determine the association of serum HE4 levels in DM patients with MOCA score, multivariate linear regression analyses were performed (Table 3). Model 1 indicated that higher serum HE4 levels were significantly associated with MOCA score after no adjustment. After adjusting for age, gender, BMI, current smoker, current drinker, admission systolic and diastolic BP and CVD history, the results of Model 2 were similar to those of Model 1. This association remained statistically significant and changed minimally after adding laboratory measurements to Model 2 (Model 3).

\section{Cox Proportional Hazard Analyses of Cognitive Decline Prediction in 205 Patients with DM}

All included DM patients $(\mathrm{N}=205)$ were prospectively followed up for a median period of 48 months (range $=5-49$ ). Cognitive decline (MCI) occurred in 47 of the included DM patients. To determine the risk factors for cognitive decline, univariate and multivariate Cox proportional hazard regression analyses were performed (Table 4). Multivariate Cox proportional hazard analysis revealed that HE4 $(\mathrm{HR}=2.408$, 95\% CI 1.669-5.238, $P<0.001)$ was an independent prognostic factor for cognitive decline after adjustment for age, gender, BMI, current smoker, current drinker, admission systolic and diastolic BP, CVD history and laboratory measurements. Kaplan-Meier analysis demonstrated that DM patients with serum HE4 levels above the mean had a significantly higher rate of MCI events than patients with serum HE4 levels below the mean value (Log rank test, $P<0.001$, data not shown).

We performed an additional sensitivity analysis to evaluate the association of serum HE4 with cognitive

Table I Baseline Characteristics of Patients with DM and Control Subjects

\begin{tabular}{|l|l|l|l|}
\hline & Control Subjects $(\mathbf{n}=1$ 196) & Patients with DM (n=205) & $P$ value \\
\hline Age (years) & $64.7(59.6-75.3)$ & $65.6(60.3-76.0)$ & 0.213 \\
Gender (male), n (\%) & $102(48.1)$ & $99(48.3)$ & 0.847 \\
HE4 (pmol/L) & $54.6 \pm 12.4$ & $96 \pm 15.8$ & $<0.001$ \\
\hline
\end{tabular}

Abbreviations: DM, diabetes mellitus; HE4, human epididymal protein 4. 
Table 2 Clinical Characteristics in 205 DM Patients at Baseline

\begin{tabular}{|l|l|}
\hline Variables & All DM Patients (n=205) \\
\hline Age (years) & $65.6(60.3-76.0)$ \\
Gender (male), n (\%) & $99(48.3)$ \\
BMI (kg/m2) & $27.1(24.5-28.6)$ \\
Current smoker, $n$ (\%) & $42(20.5)$ \\
Current drinker, $\mathrm{n}(\%)$ & $58(28.3)$ \\
Admission systolic BP (mmHg) & $134.8(121.7-152.6)$ \\
Admission diastolic BP (mmHg) & $86.5(81.6-95.8)$ \\
DM treatment, $\mathrm{n}(\%)$ & $195(95.1)$ \\
MOCA score & $25.4 \pm 3.2$ \\
Cognitive decline, $\mathrm{n}(\%)$ & $47(22.9)$ \\
\hline CVD history & \\
Hypertension, $\mathrm{n}(\%)$ & $144(70.2)$ \\
Coronary heart disease, $\mathrm{n}(\%)$ & $39(19.0)$ \\
Stroke, $\mathrm{n}(\%)$ & $37(18.0)$ \\
Others, $\mathrm{n}(\%)$ & $8(3.9)$ \\
\hline Laboratory measurements & \\
eGFR (mL/min/l.73 m²) & $57.4(55.2-59.3)$ \\
Hs-CRP (pg/mL) & $6.4(2.6-12.5)$ \\
Hb (g/L) & $105(91-119)$ \\
ALB (g/L) & $35.7(33.4-45.8)$ \\
FBG (mmol/L) & $12.4 \pm 2.1$ \\
HbAlc (\%) & $6.9(5.9-8.3)$ \\
HDL (mmol/L) & $1.3 \pm 0.20$ \\
LDL (mmol/L) & $2.54 \pm 1.01$ \\
HE4 (pmol/L) & $54.6 \pm 12.4$ \\
\hline
\end{tabular}

Notes: Data are presented as mean \pm SD for normally distributed data, as median (interquartile range) for nonnormally distributed data, and as $n$ (\%) for categoric variables.

Abbreviations: DM, diabetes mellitus; MOCA, montreal cognitive assessment; BMI, body mass index; BP, blood pressure; CVD, cardiovascular disease; eGFR, estimated glomerular filtration rate; $\mathrm{Hb}$, hemoglobin; hs-CRP, hs-C-reactive protein; ALB, albumin; FBG, fasting blood glucose on admission; HbAlc, glycosylated hemoglobin; HDL, high-density lipoprotein; LDL, low-density lipoprotein; HE4, human epididymal protein 4.

decline in DM patients by adding DM treatment as a covariate (Table 5). The sensitivity analysis showed that higher HE4 levels were still independently associated with

Table 3 The Association Between the HE4 and MOCA Score in 205 Patients with DM by Multivariate Linear Regression Analysis

\begin{tabular}{|c|l|l|l|l|}
\hline Variables & $\mathbf{R}^{\mathbf{2}}$ & $\mathbf{S \beta}$ & $\mathbf{9 5 \%} \mathbf{~ C l}$ & $\mathbf{P}$ value \\
\hline HE4 (pmol/L) & & & & \\
Model I & 0.044 & $-0.16 \mathrm{I}$ & $-0.215--0.094$ & $<0.00 \mathrm{I}$ \\
Model 2 & 0.062 & -0.152 & $-0.186--0.087$ & $<0.00 \mathrm{I}$ \\
Model 3 & 0.153 & -0.120 & $-0.15 \mathrm{I}--0.069$ & $<0.00 \mathrm{I}$ \\
\hline
\end{tabular}

Notes: Model I: No adjustment. Model 2: Adjusted for age, gender, BMI, current smoker, current drinker, admission systolic and diastolic BP and CVD history. Model 3: Adjusted for age, gender, BMI, current smoker, current drinker, admission systolic and diastolic BP, CVD history and laboratory measurements.

Abbreviations: HE4, human epididymal protein 4; DM, diabetes mellitus; MOCA, montreal cognitive assessment; BMI, body mass index; BP, blood pressure. a higher risk of cognitive decline. Stratified analysis also showed that the significant association between serum HE4 levels and MCI (end points) in DM patients was not affective by CVD history (Table 6).

\section{Discussion}

Our results in this study suggested that Multivariate Cox proportional hazard analysis suggested that HE4 was an independent prognostic factor for cognitive decline in DM patients.

In our study, we first showed that serum HE4 had a close association with MOCA score at baseline and cognitive decline after a follow-up of 4 years, which may be partly or mostly explained by the mechanistic research performed in previous studies. ${ }^{2-24}$ These studies have demonstrated that chronic vascular inflammation caused by DM promotes events of cognitive decline. ${ }^{22-24}$ The increased HE4 levels may be the result of the aggravation of inflammation in DM patients, which suggested that higher serum HE4 levels were associated with cognitive decline in DM patients. Although previous studies have proven that HE4 is not overexpressed in normal or abnormal cardiovascular tissues. ${ }^{25,26}$ Increased HE4 levels are not mainly attributable to pathological changes in cardiovascular (CV) tissue itself. The mechanism of chronic inflammation may be a better explanation of increased HE4, which is consistent with our hypothesis and results. Additionally, some studies have suggested that increased HE4 levels are closely related to renal dysfunction. ${ }^{27-29}$ The eGFR of our DM patients was normal, so the influence of renal function abnormalities on our results was excluded. Studies have also reported that HE4 is associated with cancer (ovarian, cervical, lung, breast, etc.) and some other serious diseases. ${ }^{9,10}$ To eliminate the impact of the diseases on this study, DM patients with these diseases were excluded from the baseline assessment. It is of great clinical significance to determine the independent risk factors or predictors of cognitive decline in DM patients. The early detection of cognitive decline in these patients may provide an opportunity to develop strategies aiming to reduce the medical burden and improve prognosis. Our results seemed to imply that serum HE4 might be a highly sensitive biomarker for the early recognition of cognitive decline in DM patients.

This study has some notable strengths. We first found that serum HE4 can be used as an effective prognostic factor for cognitive decline in patients with DM, and the serum test is simple and convenient for patients. Additionally, the study population exhibited a broad spectrum of risks, including primary and secondary prevention populations. 
Table 4 Cox Proportional Hazard Analysis for Predicting Cognitive Decline in 205 Patients with DM

\begin{tabular}{|l|l|l|l|}
\hline Variables & Model I & Model 2 & Model 3 \\
\hline HE4 (pmol/L) & & & \\
Quartile I & 1.000 (ref.) & 1.000 (ref.) & 1.000 (ref.) \\
Quartile 2 & $1.912(1.301-3.610)$ & $1.752(1.213-3.105)$ & $1.578(1.227-3.001)$ \\
Quartile 3 & $2.350(1.632-4.14 I)$ & $2.015(1.423-3.901)$ & $1.816(1.335-3.214)$ \\
Quartile 4 & $2.761(2.010-6.042)$ & $2.672(1.864-5.548)$ & $2.408(1.669-5.238)$ \\
\hline P-trend & $<0.001$ & $<0.001$ & $<0.001$ \\
\hline
\end{tabular}

Notes: Model I: No adjustment. Model 2: Adjusted for age, gender, BMI, current smoker, current drinker, admission systolic and diastolic BP and CVD history. Model 3: Adjusted for age, gender, BMI, current smoker, current drinker, admission systolic and diastolic BP, CVD history and laboratory measurements.

Abbreviations: HE4, human epididymal protein 4; DM, diabetes mellitus; MOCA, montreal cognitive assessment; BMI, body mass index; BP, blood pressure.

Table 5 Cox Proportional Hazard for Predicting Cognitive Decline in 205 Patients with DM by Sensitivity Analysis

\begin{tabular}{|l|l|l|l|}
\hline Variables & Model I & Model 2 & Model 3 \\
\hline $\begin{array}{l}\text { HE4 (pmol/L) } \\
\text { Quartile I }\end{array}$ & 1.000 (ref.) & 1.000 (ref.) & 1.000 (ref.) \\
Quartile 2 & $1.70 I(1.270-3.408)$ & $1.522(1.202-2.83 \mathrm{I})$ & $1.349(1.192-2.624)$ \\
Quartile 3 & $2.132(1.420-3.740)$ & $1.841(1.318-3.542)$ & $1.605(1.227-3.144)$ \\
Quartile 4 & $2.570(1.972-4.135)$ & $2.347(1.749-3.946)$ & $2.219(1.603-3.617)$ \\
\hline P-trend & $<0.001$ & $<0.001$ & $<0.001$ \\
\hline
\end{tabular}

Notes: Model I: DM treatment. Model 2: Adjusted for age, gender, BMI, current smoker, current drinker, admission systolic and diastolic BP, CVD history and DM treatment. Model 3: Adjusted for age, gender, BMI, current smoker, current drinker, admission systolic and diastolic BP, CVD history, laboratory measurements and DM treatment. Abbreviations: HE4, human epididymal protein 4; DM, diabetes mellitus; MOCA, montreal cognitive assessment; BMI, body mass index; BP, blood pressure.

Table 6 Cox Proportional Hazard for Predicting Cognitive Decline in 208 Patients with DM by Stratified Analysis

\begin{tabular}{|c|c|c|c|}
\hline Variables & Model I & Model 2 & Model 3 \\
\hline \multicolumn{4}{|l|}{ CVD history } \\
\hline HE4 (per I-SD increase) & $2.247(1.640-3.703)$ & $2.04 I(1.526-3.52 I)$ & $1.812(1.446-3.109)$ \\
\hline$P$ value & $<0.001$ & $<0.001$ & $<0.001$ \\
\hline \multicolumn{4}{|l|}{ No CVD history } \\
\hline HE4 (per I-SD increase) & $1.993(1.375-3.534)$ & $1.748(1.300-3.217)$ & 1.538 (1.206-2.994) \\
\hline$P$ value & 0.004 & 0.009 & 0.017 \\
\hline
\end{tabular}

Notes: Model I: No adjustment. Model 2: Adjusted for age, gender, BMI, current smoker, current drinker, admission systolic and diastolic BP and CVD history. Model 3: Adjusted for age, gender, BMI, current smoker, current drinker, admission systolic and diastolic BP, CVD history and laboratory measurements.

Abbreviations: HE4, human epididymal protein 4; DM, diabetes mellitus; MOCA, montreal cognitive assessment; BMI, body mass index; BP, blood pressure.

We ensured comprehensive follow-up and rigorous adjudication of CV events. Additionally, the HE4 assay chosen for this analysis is both widely available and analytically stable (coefficient of variation of precision $<5 \%$ ).

\section{Limitations}

The limitation of the study is the small sample size. More studies must be performed to identify the value of serum HE4 for predicting cognitive decline. Given the close relationships between serum HE4 and many malignant tumors or renal function, the results of this study are not applicable to the general population.

\section{Conclusions}

Serum HE4 is an independent prognostic factor for predicting cognitive decline in patients with DM. Serum HE4 might enable the early recognition of DM patients at risk of developing senile dementia.

\section{Funding}

There is no funding to report.

\section{Disclosure}

The authors report no conflicts of interest for this work. 


\section{References}

1. Kukull WA, Higdon R, Bowen JD, et al. Dementia and Alzheimer disease incidence: a prospective cohort study. Arch Neurol. 2002;59:1737-1746. doi:10.1001/archneur.59.11.1737

2. Akomolafe A, Beiser A, Meigs JB, et al. Diabetes mellitus and risk of developing Alzheimer disease: results from the Framingham Study. Arch Neurol. 2006;63(11):1551-1555. doi:10.1001/archneur.63.11.1551

3. Wrighten SA, Piroli GG, Grillo CA, et al. A look inside the diabetic brain: contributors to diabetes-induced brain aging. Biochim Biophys Acta. 2009;1792:444-453.

4. Ott A, Stolk RP, van Harskamp F, et al. Diabetes mellitus and the risk of dementia: the Rotterdam Study. Neurology. 1999;53:1937-1942. doi:10.1212/WNL.53.9.1937

5. Biessels GJ, Staekenborg S, Brunner E, et al. Risk of dementia in diabetes mellitus: a systematic review. Lancet Neurol. 2006;5:64-74. doi:10.1016/S1474-4422(05)70284-2

6. Ghezzi P, Floridi L, Boraschi D, et al. Oxidative Stress and Inflammation Induced by Environmental and Psychological Stressors: A Biomarker Perspective. Antioxid Redox Signal. 2018;28(9):852-872.

7. Kirchhoff C, Habben I, Ivell R, et al. A major human epididymisspecific cDNA encodes a protein with sequence homology to extracellular proteinase inhibitors. Biol Reprod. 1991;45(2):350-357. doi:10.1095/biolreprod45.2.350

8. Kirchhoff C. Molecular characterization of epididymal proteins. Rev Reprod. 1998;3(2):86-95.

9. Hellstrom I, Raycraft J, Hayden-Ledbetter M, et al. The HE4 (WFDC2) protein is a biomarker for ovarian carcinoma. Cancer Res. 2003;63(13):3695-3700.

10. Plebani M. HE4 in gynecological cancers: report of a European investigators and experts meeting. Clin Chem Lab Med. 2012;50 (12):2127-2136. doi:10.1515/cclm-2012-0373

11. Hertlein L, Stieber P, Kirschenhofer A, et al. Human epididymis protein 4 (HE4) in benign and malignant diseases. Clin Chem Lab Med. 2012;50(12):2181-2188. doi:10.1515/cclm-2012-0097

12. Galgano MT, Hampton GM, Frierson HJ. Comprehensive analysis of HE4 expression in normal and malignant human tissues. Mod Pathol. 2006;19(6):847-853.

13. Bingle CD, Vyakarnam A. Novel innate immune functions of the whey acidic protein family. Trends Immunol. 2008;29(9):444-453. doi:10.1016/j.it.2008.07.001

14. Escudero JM, Auge JM, Filella X, et al. Comparison of serum human epididymis protein 4 with cancer antigen 125 as a tumor marker in patients with malignant and nonmalignant diseases. Clin Chem. 2011;57(11):1534-1544. doi:10.1373/clinchem.2010.157073

15. Drapkin R, von Horsten HH, Lin Y, et al. Human epididymis protein 4 (HE4) is a secreted glycoprotein that is overexpressed by serous and endometrioid ovarian carcinomas. Cancer Res. 2005;65(6):21622169. doi:10.1158/0008-5472.CAN-04-3924
16. Yaffe K, Kanaya A, Lindquist K, et al. The metabolic syndrome, inflammation, and risk of cognitive decline. JAMA. 2004;292 (18):2237. doi:10.1001/jama.292.18.2237

17. Schram MT, Euser SM, Craen AJD, et al. Systemic markers of inflammation and cognitive decline in old age. Alzheimers Dement. 2006;2(3-supp-S):S154. doi:10.1016/j.jalz.2006.05.575

18. Bucht G, Adolfsson R, Lithner F, et al. Changes in blood glucose and insulin secretion in patients with senile dementia of alzheimer type. Acta Med Scand. 2010;213(5):387-392. doi:10.1111/j.09546820.1983.tb03756.x

19. Sundar U, Sharma A, Yeolekar ME. Presenile dementia-etiology, clinical profile and treatment response at four month follow up. $J$ Assoc Physicians India. 2004;52:953-958.

20. Alberti KG, Zimmet PZ. New diagnostic criteria and classification of diabetes-again? Diabet Med. 2015;15(7):535-536. doi:10.1002/ (SICI)1096-9136(199807)15:7<535::AID-DIA670>3.0.CO;2-Q

21. Levey AS, Stevens LA, Schmid $\mathrm{CH}$, et al. A new equation to estimate glomerular filtration rate. Ann Intern Med. 2009;150 (9):604-612. doi:10.7326/0003-4819-150-9-200905050-00006

22. Chen Q, Mo R, Wu N, et al. Berberine ameliorates diabetes-associated cognitive decline through modulation of aberrant inflammation response and insulin signaling pathway in DM rats. Front Pharmacol. 2017;8:334. doi:10.3389/fphar.2017.00334

23. Tian X, Liu Y, Ren G, et al. Resveratrol limits diabetes-associated cognitive decline in rats by preventing oxidative stress and inflammation and modulating hippocampal structural synaptic plasticity. Brain Res. 2016;1650:1-9.

24. Liu YW, Zhu X, Yang QQ, et al. Suppression of methylglyoxal hyperactivity by mangiferin can prevent diabetes-associated cognitive decline in rats. Psychopharmacology. 2013;228(4):585-594. doi:10.1007/s00213-013-3061-5

25. De Boer RA, Cao Q, Postmus D, et al. The WAP four-disulfide core domain protein HE4: a novel biomarker for heart failure. JACC Heart Fail. 2013;1(2):164-169. doi:10.1016/j.jchf.2012.11.005

26. Piek A, Meijers WC, Schroten NF, Gansevoort RT, de Boer RA, Silljé HH. HE4 serum levels are associated with heart failure severity in patients with chronic heart failure. J Card Fail. 2017;23(1):12-19. doi:10.1016/j.cardfail.2016.05.002

27. Yuan T, Li Y. Human epididymis protein 4 as a potential biomarker of chronic kidney disease in female patients with normal ovarian function. Lab Med. 2017;48(3):238-243. doi:10.1093/labmed/lmx036

28. Huang Y, Jiang H, Zhu L. Human epididymis protein 4 as an indicator of acute heart failure in patients with chronic kidney disease. Lab Med. 2020;51(2):169-175.

29. Nagy B Jr, Krasznai ZT, Balla H, et al. Elevated human epididymis protein 4 concentrations in chronic kidney disease. Ann Clin Biochem. 2012;49(Pt4):377-380. doi:10.1258/acb.2011.011258

\section{Publish your work in this journal}

Diabetes, Metabolic Syndrome and Obesity: Targets and Therapy is an international, peer-reviewed open-access journal committed to the rapid publication of the latest laboratory and clinical findings in the fields of diabetes, metabolic syndrome and obesity research. Original research, review, case reports, hypothesis formation, expert opinion and commentaries are all considered for publication. The manuscript management system is completely online and includes a very quick and fair peer-review system, which is all easy to use. Visit http://www.dovepress.com/testimonials.php to read real quotes from published authors. 